\title{
Profil lipidique, cinétique d'apparition des diènes conjugués et activité paraoxonase- 1 chez le diabétique de type 2 à Libreville au Gabon
}

\author{
Félix Ovono ABESSOLO*, Serge BEKALE, Fernandez JOSE, \\ Yangou MOUTSINGA et Edouard Ngou MILAMA \\ Département de Chimie Biochimie, Faculté de Médecine, \\ Université des Sciences de la Santé, BP 4009 Libreville, Gabon. \\ *Auteur correspondant, E-mail : ovonab@yahoo.fr
}

\section{RESUME}

L'objectif de ce travail était d'identifier la fréquence des dyslipidémies du diabétique de type 2 afin d'apprécier l'efficacité de leur prise en charge. Pour cela, une étude prospective et ponctuelle, a été menée du $1^{\text {er }}$ janvier au 30 octobre 2010. Elle a concerné les patients diabétiques de type 2, avec ou sans complications macro et microvasculaires. Un bilan lipidique a été réalisé chez ces patients à jeun depuis 12 heures, associé à la détermination du temps de latence d'apparition des diènes conjugués et l'activité paraoxonase-1 (PON1). Il a été démontré que le LDL était élevé chez 65,2\%, alors que le HDL était réduit chez 63,6\% des diabétiques. Le profil le plus athérogène, caractérisé par une augmentation du LDL et une diminution du HDL intéressait $31,8 \%$ des sujets. La dyslipidémie classiquement associée au diabète de type 2, n'était retrouvée que chez 8,3\% des patients. Ces anomalies quantitatives étaient accompagnées de modifications qualitatives des lipides, à type de diminution du temps de latence d'apparition des diènes conjugués ou de l'activité PON1 avec la chronicité de la maladie. Ces résultats suggèrent l'amélioration des mesures de prise en charge des dyslipidémies chez les diabétiques de type 2, non seulement avec les statines, mais également les dérivés de l'acide nicotinique et même des antioxydants comme la vitamine $\mathrm{E}$.

(C) 2012 International Formulae Group. All rights reserved.

Mots clés : diabète de type 2, dyslipidémies, athérosclérose, oxydation LDL, paraoxonase 1.

\section{INTRODUCTION}

Le diabète est actuellement considéré comme une épidémie métabolique (Hall et al., 2011). Sa prévalence estimée en 2010 était de 221 millions dans le monde (Simon et al., 2005). Cette épidémie touche non seulement les pays développés, mais également les pays africains. C'est ainsi qu'au Gabon, la prévalence de cette maladie dans la population générale variait de 0,3 en 1990 à $0,7 \%$ en 1994 (Ntyonga-Pono et Nguemby, 1996). Le diabète de type 2, correspondant à plus de 90\% des diabètes, représente l'essentiel du problème de santé publique constitué par cette épidémie (Hall et al., 2011). Il est associé à une morbidité et une mortalité importantes. L'atteinte vasculaire domine la pathogenèse de ses complications parmi lesquelles la macroangiopathie est la plus commune et la plus sévère, car plus de $80 \%$ des diabétiques de type 2, vont en présenter au cours de l'évolution de leur maladie (Boutouyriel et al., 
2005). En tenant compte de ces complications, la prise en charge du risque cardiovasculaire est devenue une nécessité chez les diabétiques. En effet, la modification des facteurs de risque cardiovasculaire par le traitement de l'hypertension artérielle ou encore par l'utilisation des agents pharmacologiques diminuant la concentration des lipides, est devenue primordiale et pourrait être plus efficace et moins coûteuse, que le traitement de l'hyperglycémie ellemême (Vijian et Hayward, 2003). Plusieurs études ont effectivement mis en évidence des perturbations du métabolisme des lipides chez le sujet diabétique de type 2, avec classiquement une diminution du cholestérol HDL, une augmentation des triglycérides et des particules de cholestérol LDL denses et petites, qui sont les plus athérogènes (Ginsberg et MacCallum, 2009). Ce profil lipidique est à l'origine de l'utilisation des hypolipémiants chez le sujet diabétique de type 2. Au Gabon, une évaluation de la prise en charge de ces complications métaboliques, par l'étude du profil lipidique des sujets atteints de diabète de type 2 a été entreprise.

\section{MATERIEL ET METHODES}

Il s'agissait d'une étude prospective et ponctuelle, réalisée du $1^{\text {er }}$ janvier au 30 octobre 2010 à Libreville au Gabon. Le recrutement a été effectué dans le service d'endocrinologie du Centre Hospitalier de Libreville et au Laboratoire de Biochimie de la Faculté de Médecine, à l'Université des Sciences de la Santé de la même ville. Tout patient diabétique de type 2 connu et traité en ambulatoire, reçut pour le contrôle de son diabète, était recruté. Les sujets atteints de diabète de type 1 , de diabète gestationnel ou de diabète secondaire et ceux qui refusaient de signer l'accord écrit étaient exclus. Ce protocole a été réalisé selon les recommandations de la déclaration d'Helsinki sur la recherche avec les êtres humains, révisée en 2004 (World Medical Association, 2004).
L'indice de masse corporelle (IMC) a été calculé en divisant le poids (en $\mathrm{kg}$ ) par le carré de la taille en mètres. Le déficit pondéral était défini par un IMC inférieur à $18,5 \mathrm{~kg} / \mathrm{m}^{2}$. Le poids normal correspondait à un IMC qui variait de $18,5-24,9 \mathrm{~kg} / \mathrm{m}^{2}$, alors que l'obésité correspondait à un IMC supérieur ou égal à 30 $\mathrm{kg} / \mathrm{m}^{2}$. Les sujets hypertendus étaient ceux qui suivaient un traitement anti hypertenseur ou ceux dont la pression artérielle systolique et/ou la diastolique était supérieure à 130 et 80 $\mathrm{mmHg}$ respectivement (Vijian et Hayward, 2003). La macroangiopathie était définie par l'apparition dans l'histoire de la maladie, d'un évènement cardiovasculaire et/ou la présence d'un angor de poitrine, d'une claudication intermittente des membres inférieurs, l'abolition des pouls périphériques ou des anomalies ischémiques à l'électrocardiogramme, notifiés dans le carnet de suivi. La neuropathie périphérique était retrouvée à l'examen clinique: réflexes rotulien et achilléen, des para et/ou des diestésies. La rétinopathie était recherchée dans le carnet de suivi du diabétique. La néphropathie correspondait à la présence d'une protéinurie à la bandelette urinaire ou un débit de filtration glomérulaire inférieur ou égal à $90 \mathrm{ml} / \mathrm{min}$ obtenu à partir de la formule de Cockcroft et Gault.

Une concentration de cholestérol total supérieure à 5,2 $\mathrm{mmol} / \mathrm{l}$, un LDL supérieur à 2,6 $\mathrm{mmol} / \mathrm{l}$ et des triglycérides supérieurs à $1,7 \mathrm{mmol} / \mathrm{l}$ étaient considérés comme élevés, alors que les concentrations de HDL inférieures à 1,04 mmol/l étaient qualifiées de basses. A partir de ces valeurs limites, trois groupes de patients ont été identifiés. Les patients présentant une hypercholestérolémie étaient définis par un cholestérol LDL supérieur à 2,6 mmol/l et une triglycéridémie inférieure à $1,7 \mathrm{mmol} / \mathrm{l}$. Les sujets ayant une hypertriglycéridémie étaient ceux qui avaient une concentration des triglycérides supérieure à $1,7 \mathrm{mmol} / \mathrm{l}$ et une concentration des LDL inférieure à 2,6 $\mathrm{mmol} / \mathrm{l}$. La dyslipidémie mixte était définie par des concentrations de 
LDL et triglycérides supérieures à 2,6 mmol/1 et $1,7 \mathrm{mmol} / \mathrm{l}$ respectivement. La dyslipidémie $\mathrm{du}$ sujet diabétique correspondait à une concentration des HDL inférieure à 1,04 $\mathrm{mmol} / \mathrm{l}$ associée à une hypertriglycéridémie.

Le dosage de la glycémie a été effectué par la méthode à la glucose oxydase. Le cholestérol total et les triglycérides ont été dosés par les méthodes enzymatiques classiques. La fraction de cholestérol HDL était obtenue après précipitation des autres fractions par le tungstate de magnésium et suivi du dosage du cholestérol sur le surnageant. Le cholestérol LDL était obtenu par la formule de Friedewald pour les échantillons dont la concentration des triglycérides était inférieure à 4,6 $\mathrm{mmol} / \mathrm{l}$ (Rijks, 1995). L'extraction de la fraction du cholestérol LDL dense et petite (sdLDL) était basée sur la méthode proposée par Hirano et al. (2003). A $100 \mu \mathrm{l}$ d'une solution de précipitation constituée d'héparine sodique (150 U/ml) et de $\mathrm{MgCl}_{2}(90 \mathrm{mmol} / \mathrm{l})$, on a ajouté $100 \mu \mathrm{l}$ de sérum. Le mélange a été passé au vortex puis incubé à $37{ }^{\circ} \mathrm{C}$ pendant 10 minutes. Ce mélange a ensuite été transféré dans un bain de glace pendant 15 minutes. Après cette seconde incubation, les échantillons ont été centrifugés à 15.000 tours/min pendant 15 minutes à $4{ }^{\circ} \mathrm{C}$. Le surnageant obtenu contenait le sdLDL. Les sdLDL ont été oxydés par la méthode de Esterbauer et al. (1989). A $100 \mu \mathrm{l}$ du surnageant obtenu précédemment, ont été ajoutés $320 \mu \mathrm{l}$ d'une solution de $\mathrm{CuCl}_{2} 1$ mmol/l. L'apparition des produits d'oxydation qui sont des diènes conjugués, a été suivie par spectrophotométrie à $234 \mathrm{~nm}$ (Emma et al., 2000). Pour évaluer l'activité paraoxonase-1, nous avons préparé $250 \mu \mathrm{l}$ d'un tampon Tris$\mathrm{HCl} 20 \mathrm{mmol} / \mathrm{l}, \mathrm{pH} 8$ contenant du phénylacétate $(1 \mathrm{mmol} / \mathrm{l})$ et $\mathrm{du} \mathrm{CaCl}_{2} \quad(2$ $\mathrm{mmol} / \mathrm{l})$. Nous y avons rajouté $100 \mu \mathrm{l}$ de sérum. La réaction a été suivie par spectrophotométrie à $270 \mathrm{~nm}$. Le coefficient d'extinction molaire moléculaire à cette longueur d'onde était de $1307 \mathrm{~mol} / \mathrm{l} / \mathrm{cm}$. Une unité d'activité arylestérase correspondait à 1 $\mu \mathrm{mol}$ de phénylacétate hydrolysée par ml et par minute (De Geest et al., 2000).

\section{Analyse statistique}

Les estimateurs utilisés étaient la moyenne et son écart type pour les variables quantitatives et la fréquence pour les variables qualitatives. L'association entre ces deux types de paramètres était étudiée par le test non paramétrique de Kruskal Wallis. L'étude des corrélations entre variables quantitatives a été faite par le Rho de Spearman. Les différences étaient considérées comme significatives pour $\mathrm{p}<0,05$.

\section{RESULTATS}

L'échantillon de ce travail était constitué de 528 patients diabétiques, dont 284 femmes $(53,8 \%)$ et 244 hommes $(46,2 \%)$, soit un sex ratio de 0,86 . L'âge moyen de ces patients était de 53,3 $\pm 10,9$ ans, avec des extrêmes allant de 31 à 84 ans, sans différence significative entre les femmes et les hommes $(\mathrm{p}=0,8307)$. La concentration moyenne $\mathrm{du}$ cholestérol total était de 5,2 $\pm 1,6 \mathrm{mmol} / \mathrm{l}$ (Tableau 1). Elle était plus élevée chez les sujets du genre féminin 5,6 $\pm 1,6 \mathrm{mmol} / \mathrm{l}$, contre 4,8 $\pm 1,5 \mathrm{mmol} / \mathrm{l}$ pour les hommes, avec une différence significative $(\mathrm{p}=0,0047)$. Par contre, il n'y avait pas de différence statistiquement significative entre les fumeurs et les non fumeurs $(5,2 \pm 1,6 \mathrm{mmol} / \mathrm{l}$ versus $4,3 \pm 2,1 \mathrm{mmol} / \mathrm{l} ; \mathrm{p}=0,1428)$. L'apparition d'une complication chronique n'avait pas de lien avec la cholestérolémie. En effet, la concentration de cholestérol total était à 5,2 \pm 1,6 $\mathrm{mmol} / \mathrm{l}$ chez les sujets atteints de neuropathie, versus $5,2 \pm 1,8 \mathrm{mmol} / \mathrm{l}$ pour les sujets atteints de diabète non compliqué. De même, en cas d'apparition d'une néphropathie, les concentrations de cholestérol total étaient de 4,6 $\pm 1,7 \mathrm{mmol} / \mathrm{l}$, contre 5,3 $\pm 1,7 \mathrm{mmol} / \mathrm{l}$, sans que toutes ces différences soient statistiquement significatives. Par ailleurs, les concentrations de cholestérol total n'étaient pas différentes 
selon que les sujets étaient hypertendus ou non (Tableau 2).

La concentration moyenne de triglycérides dans cet échantillon était de 2,0 \pm $0,9 \mathrm{mmol} / \mathrm{l}$. Cette concentration n'était pas statistiquement différente selon le genre : de $1,32 \pm 0,5 \mathrm{mmol} / \mathrm{l}$ chez les hommes, elle était à $1,05 \pm 0,3 \mathrm{mmol} / \mathrm{l}$ chez les femmes $(\mathrm{p}=$ 0,1373). Les triglycérides étaient significativement abaissés chez les diabétiques pratiquant du sport : $0,84 \pm 0,3$ $\mathrm{mmol} / \mathrm{l}$, contre $1,2 \pm 0,4 \mathrm{mmol} / \mathrm{l}(\mathrm{p}=0,0198)$. Une augmentation des triglycérides a été observée, chez les fumeurs et les diabétiques qui avouaient grignoter entre les repas. Par contre, la consommation d'alcool ne semblait pas modifier les concentrations des triglycérides dans cette étude sur le sujet diabétique.

La concentration moyenne de cholestérol HDL était de 1,5 $\pm 0,3 \mathrm{mmol} / \mathrm{l}$, dans toute la population de diabétiques. Ce cholestérol HDL était plus élevé chez les femmes $(1,7 \pm 0,7 \mathrm{mmol} / \mathrm{l})$ que chez les hommes $(1,3 \pm 0,8 \mathrm{mmol} / \mathrm{l})$ avec cependant de fortes variations selon les individus $(\mathrm{p}=$ 0,0356). Ni la consommation de tabac, ni celle de l'alcool, ne semblaient faire varier cette concentration du cholestérol des HDL ( $\mathrm{p}=$ 0,7818 et $p=0,8408$ respectivement). La concentration moyenne du cholestérol LDL était de 3,2 $\pm 0,9 \mathrm{mmol} / \mathrm{l}$. Ce cholestérol LDL était à $3,3 \pm 1,1 \mathrm{mmol} / 1 \mathrm{chez}$ les femmes et 3,1 $\pm 0,5 \mathrm{mmol} / \mathrm{l}$ chez les hommes, sans que cette différence ne soit statistiquement significative $(\mathrm{p}=0,1624)$. Une augmentation de la concentration de cholestérol LDL a été notée chez les sujets consommant de l'alcool. Alors que le tabac n'avait pas d'influence sur cette fraction de cholestérol. En outre, des corrélations positives entre l'indice de masse corporelle et le cholestérol total $(r=0,367)$ d'une part, et le cholestérol HDL $(r=0,307)$ d'autre par,t ont été observées. Les anomalies lipidiques isolées les plus fréquentes étaient une augmentation du LDL $(65,2 \%)$ et une diminution du cholestérol HDL (63,6\%). L'augmentation des triglycérides n'était objectivée que chez $16,7 \%$ de tous les diabétiques de ce travail. Pour ce qui était des perturbations associées, le profil le plus athérogène, caractérisé par une augmentation du LDL et des triglycérides, associée à une diminution du cholestérol HDL était obtenu chez 7,0\% des diabétiques. La dyslipidémie du sujet diabétique, dans laquelle on retrouvait une hypertriglycéridémie et une diminution du cholestérol HDL, n'était retrouvée que chez $8,3 \%$ des patients. La dyslipidémie mixte concernait $12,1 \%$ des sujets de cette étude (Tableau 3).

Le temps de latence d'apparition des diènes conjugués était plus élevé chez les sujets diabétiques sans complications. La complication qui était la plus associée à une diminution du temps d'apparition des diènes conjugués était la macroangiopathie $(67 \pm 2$ s), alors que la rétinopathie correspondait à un temps de $73 \pm 4$ s et l'hypertension artérielle était associée à un temps de $71 \pm 6 \mathrm{~s}$. Par contre, les sujets atteints de néphropathie montraient un temps de latence de $81 \pm 3$ s.

L'activité paraoxonase-1 était distribuée de la même manière, malgré la grande variabilité d'activités. En effet, on retrouvait une activité plus importante chez les diabétiques sans complications $(0,42 \pm 0,11$ $\mathrm{mUI} / \mathrm{ml}$ ) et chez les sujets atteints de néphropathie $(0,38 \pm 0,05 \mathrm{mUI} / \mathrm{ml})$. Les patients atteints d'autres complications avaient des activités enzymatiques nettement plus faibles, avec $0,24 \pm 0,06 \mathrm{mUI} / \mathrm{ml}$ pour les sujets ayant une macroangiopathie, 0,25 \pm $0,08 \mathrm{mUI} / \mathrm{ml}$ pour ceux qui avaient une rétinopathie et $0,28 \pm 0,16 \mathrm{mUI} / \mathrm{ml}$ pour les diabétiques ayant une hypertension artérielle (Tableau 4). 
Tableau 1 : Paramètres moyens de la population d'étude.

\begin{tabular}{lcc}
\hline Paramètres & Moyennes & Ecart-types \\
\hline Age (ans) & 53,3 & 10,9 \\
Poids $(\mathrm{kg})$ & 76,4 & 4,1 \\
Pression systolique $(\mathrm{mmHg})$ & 157 & 24 \\
Pression diastolique $(\mathrm{mmHg})$ & 104 & 10 \\
Indice de masse corporelle $\left(\mathrm{kg} / \mathrm{m}^{2}\right)$ & 26,5 & 6,3 \\
Temps de latence (secondes) & 74 & 4 \\
Activité PON-1 (mU/ml) & 0,32 & 0,11 \\
Cholestérol total (mmol/l) & 5,2 & 0,6 \\
Cholestérol HDL (mmol/l) & 1,5 & 0,3 \\
Cholestérol LDL(mmol/l) & 3,2 & 0,9 \\
Triglycérides (mmol/l) & 2,0 & 0,9 \\
Glycémie (mmol/l) & 8,2 & 3,0 \\
HbAlc $(\%)$ & 8,3 & 3,0 \\
Débit de filtration glomérulaire $(\mathrm{ml} / \mathrm{min})$ & 95 & 16 \\
Créatinine plasmatique (mmol/l) & 143 & 23 \\
\hline
\end{tabular}

Tableau 2: Valeurs des probabilités p des relations entre paramètres biologiques, les habitudes et modes de vie et les complications du diabète.

\begin{tabular}{lcccc}
\hline Variables & Cholestérol total & Triglycérides & HDL cholestérol & LDL cholestérol \\
\hline Alcool & 0,0225 & 0,3352 & 0,8408 & 0,0420 \\
Tabac & 0,1418 & 0,0089 & 0,7818 & 0,2245 \\
Sport & 0,884 & 0,0198 & 0,6020 & 0,9400 \\
Grignotage & 0,9893 & 0,0404 & 0,5530 & 0,9372 \\
HTA & 0,7615 & 0,1723 & 0,4063 & 0,278 \\
Neuropathie & 0,9432 & 0,1570 & 0,5731 & 0,8102 \\
Néphropathie & 0,2703 & 0,1571 & 0,7166 & 0,1415 \\
Rétinopathie & 0,9106 & 0,7088 & 0,3566 & 0,6524 \\
Macroangiopathie & 0,4637 & 0,1060 & 0,3058 & 0,7627 \\
\hline
\end{tabular}

Tableau 3 : Principaux profils athérogènes retrouvés.

\begin{tabular}{lcc}
\hline Anomalies & Nombre de cas & Pourcentages \\
\hline LDL élevé & 344 & 65,2 \\
Triglycérides élevés & 88 & 16,7 \\
HDL bas & 336 & 63,6 \\
LDL et triglycérides élevés & 64 & 12,1 \\
HDL bas et triglycérides élevés & 44 & 8,3 \\
LDL élevés, HDL bas et triglycérides élevés & 28 & 7,0 \\
LDL élevés et HDL bas & 168 & 31,8 \\
\hline
\end{tabular}


Tableau 4 : Temps de latence d'oxydation du dLDL et activités enzymatiques PON1 (moyenne + écart-type).

\begin{tabular}{lccc}
\hline Etat physiopathologique & Nombre (\%) & Oxydation LDL (s) & PON-1 (mUI/ml) \\
\hline Diabète & $120(22,7)$ & $80 \pm 4$ & $0,42 \pm 0,11$ \\
Diabète + HTA & $112(21,2)$ & $71 \pm 6$ & $0,28 \pm 0,16$ \\
Néphropathie & $100(18,9)$ & $81 \pm 3$ & $0,38 \pm 0,05$ \\
Rétinopathie & $100(18,9)$ & $73 \pm 4$ & $0,25 \pm 0,08$ \\
Macroangiopathie & $96(18,2)$ & $67 \pm 2$ & $0,24 \pm 0,06$ \\
\hline Total & $\mathbf{5 2 8}(\mathbf{1 0 0})$ & $\mathbf{7 4} \pm \mathbf{6}$ & $\mathbf{0 , 3 2} \pm \mathbf{0 , 1 1}$ \\
\hline
\end{tabular}

\section{DISCUSSION}

Les complications cardiovasculaires sont responsables du taux élevé de mortalité dans le diabète de type 2 , d'où la nécessité d'un contrôle stricte de la tension artérielle et des dyslipidémies. Dans ce cadre, nous avons entrepris d'étudier les différents profils lipidiques observés au cours du diabète de type 2 à Libreville au Gabon. Le recrutement des malades a été effectué au service d'endocrinologie du Centre Hospitalier de Libreville et au Laboratoire de Biochimie de la Faculté de Médecine et des Sciences de la Santé de Libreville. Ce sont les deux principaux sites de prise en charge clinique et biologique de ces patients au Gabon. Ont été évalués chez ces patients, outre la glycémie, l'hémoglobine glyquée HbA1C, les triglycérides, le cholestérol total et ses fractions HDL et LDL. Les limites de référence utilisées dans ce travail sont une conjonction des cibles thérapeutiques proposées par l'Association de Langue Française pour l'Etude du Diabète et des Maladies Métaboliques (ALFEDIAM) et les valeurs de référence des lipides obtenues au Laboratoire de Biochimie de la Faculté de Médecine de Libreville. L'ALFEDIAM a proposé des cibles thérapeutiques dans la prise en charge des dyslipidémies chez le sujet diabétique de type 2 (AFSSAPS, 2001). Cependant, le National Cholesterol Education Program Adult Treatment Panel III (NCEP ATP III) (Grundy et al., 2004) propose d'assimiler le diabète à un infarctus du myocarde, d'où la nécessité d'utiliser chez ces malades, les cibles thérapeutiques identiques à celles des sujets ayant déjà connu un infarctus du myocarde. La valeur cible pour le cholestérol total est de 5,2 $\mathrm{mmol} / \mathrm{l}$. Cette valeur est la même que la valeur de référence du cholestérol total, au sein de la population générale gabonaise, valeur trouvée par l'équipe de Ngou-Milama et al. (1997). Par conséquent, la valeur cible du cholestérol total utilisée dans ce travail est surestimée chez les sujets diabétiques. La valeur cible du cholestérol LDL proposée par l'ALFEDIAM est de $4,1 \mathrm{mmol} / \mathrm{l}$ ou 3,4 $\mathrm{mmol} / \mathrm{l}$ chez un patient ayant un ou plusieurs autres facteurs de risque cardio-vasculaires. Cependant, toutes les études longitudinales actuelles, de même que le NCEP, proposent une valeur cible de $2,6 \mathrm{mmol} / \mathrm{l}$, dans une population de diabétiques de type 2. C'est donc cette valeur qui a été utilisée dans cette étude. Par ailleurs, la valeur cible du cholestérol HDL utilisée est de 1,05 mmol/1 et de $1,7 \mathrm{mmol} / \mathrm{l}$ pour les triglycérides.

Il a été mis en évidence des concentrations moyennes de cholestérol à 5,6 $\pm 1,6 \mathrm{mmol} / \mathrm{l}$ et $4,8 \pm 1,5 \mathrm{mmol} / \mathrm{l}$ chez les hommes et les femmes respectivement $(\mathrm{p}=$ 0,0047). Ces valeurs sont similaires à celles obtenues dans la population générale par Ngou-Milama et al. (1997) au Gabon et Youmbissi et al. (2001) au Cameroun. La différence entre hommes et femmes observée dans ce travail, serait due à l'effet cardioprotecteur des hormones féminines, 
avant la ménopause. De plus, et contrairement aux travaux de Youmbissi et al. (2001) sur les sujets hypertendus du Cameroun, il n'y avait pas de variation de la concentration de cholestérol selon que le sujet soit hypertendu ou non. Cependant, ce résultat a été retrouvé dans d'autres études (Martin et al., 1986). Ceci pourrait s'expliquer par une association des effets conjugués de l'hypertension artérielle et du diabète dans la population étudiée. La concentration moyenne de triglycérides de cette étude est de 2,0 $\pm 0,9 \mathrm{mmol} / \mathrm{l}$; elle est largement supérieure à la limite de référence de la population saine qui est de $1,7 \mathrm{mmol} / \mathrm{l}$ (Ngou-Milama et al., 1997). Ceci renforce le constat que le diabète s'accompagne souvent d'une augmentation de la concentration des triglycérides. Les concentrations moyennes de HDL et des LDL étaient respectivement de $1,5 \pm 0,3 \mathrm{mmol} / \mathrm{l}$ et $3,3 \pm 1,1 \mathrm{mmol} / \mathrm{l}$. Ces valeurs sont caractérisées par leur variabilité, avec des écarts types importants. Elles se trouvent dans les mêmes intervalles que les concentrations de HDL et de LDL obtenues par Dehout sur une population de Bantous autochtones (Dehout et al., 2008).

Les anomalies lipidiques les plus fréquemment retrouvées dans ce travail sont l'hypercholestérolémie et la diminution du cholestérol HDL. L'hypercholestérolémie est pratiquement trois fois plus fréquente par rapport à l'étude de Marant et al. (2008) qui avaient trouvé $24 \%$ d'hypercholestérolémie chez les sujets diabétiques ayant participé à l'étude ENTRED en France. Cette proportion est de même ordre de grandeur que celle obtenue par Vezi et Naido (2005) chez les Noires en Afrique du Sud, mais supérieure à celle obtenue par Klisiewics et Raal (2009) dans toute la population de ce même pays. L'augmentation du cholestérol LDL et des triglycérides suggère une faible utilisation de thérapeutiques visant à corriger cette dyslipidémie, c'est-à-dire l'association de mesures hygiéno-diététiques et des statines (Marant et al., 2008). En effet, dans ce travail, seuls $2,1 \%$ des sujets étaient sous hypocholestérolémiants. Cependant, il est actuellement reconnu que malgré une réduction convenable de la concentration de LDL, il subsiste chez le sujet diabétique un risque résiduel inacceptablement élevé de développer une complication cardiovasculaire, d'où l'initiative des «3R » visant la réduction $\mathrm{du}$ risque résiduel (Fruchart et al., 2008). Ce risque résiduel est mis en évidence dans ce travail, où $63,6 \%$ des diabétiques ont un cholestérol HDL anormalement bas. Klisiewics et Raal (2009) en Afrique du Sud avaient trouvé 70,7\% de baisse de cholestérol HDL. Au Kénya, Otieno et al. (2005) parlaient de 72,2\% et même Okafor et al. (2008) au Nigéria avaient trouvé $88 \%$ de baisse de HDL chez les diabétiques de type 2 . Tous ces résultats vont dans le même sens, et font penser à une prise en charge peu optimale de la dyslipidémie du sujet diabétique dans ces régions. Ces sujets sont à risque cardiovasculaire particulièrement élevé. Par ailleurs, les anomalies qualitatives des lipoprotéines exprimées par le temps de latence d'apparition des diènes conjugués ou l'activité PON1, étaient associées aux complications chroniques telles que la présence de l'hypertension artérielle, la rétinopathie, contrairement à la néphropathie. L'évolution parallèle de l'activité de la paraoxonase-1 et du temps de latence, suggère que l'activité antioxydante du glucose lors de l'oxydation par des ions $\mathrm{Cu}^{2+}$ est la même dans tous les groupes étudiés et ne perturbe donc pas l'interprétation du temps de latence des diènes. Ces relations reflètent l'augmentation du risque cardiovasculaire associée à la chronicité de la maladie. La réduction de ce risque pourrait être obtenue par l'administration de molécules à activité antioxydante telle la vitamine $E$ comme le suggère Otero et al. (2005). L'hypertriglycéridémie n'a été retrouvée que dans $16,7 \%$ des cas. Ce résultat est proche de celui trouvé par plusieurs auteurs en Afrique Noire (Otero et al., 2005 ; Okafor et al., 2008; Sevum et al., 2010). 
Cependant, d'autres travaux mettent en évidence des hypertriglycéridémies chez un nombre plus important de sujets. En effet, Vezi et Naidoo (2005) avaient trouvé 57,1\% d'hypertriglycéridémie chez les Noires en Afrique du Sud, Mengesha (2006) de son côté rapportait une proportion de 38,9\% d'hypertriglycéridémie à Gaborone au Botswana. Cette disparité des résultats peut être due à de légères variations de la borne supérieure des triglycérides. Elle était en effet de 1,5 mmol/l dans l'étude de Klisiewics et Raal (2009) contre $1,7 \mathrm{mmol} / \mathrm{l}$ dans ce travail. Cependant, il semble également qu'il existe des facteurs environnementaux ayant un impact sur les concentrations des triglycérides et pouvant expliquer ces différences. En effet, Sumner et al. (2010) ont mis en évidence une hypertriglycéridémie chez seulement $25 \%$ des sujets d'Afrique de l'Ouest ayant un syndrome métabolique. De même, la récente étude de Fezeu et al. (2010) a démontré que l'urbanisation avait un impact sur les anomalies de l'obésité, dont l'hypertriglycéridémie. Il reste cependant à identifier ces facteurs environnementaux sur lesquels des mesures de prévention devraient être accentuées. Le profil caractéristique du diabétique de type 2 n'est retrouvé que chez $5,3 \%$ des sujets. De même, le profil le plus athérogène, caractérisé par une augmentation $\mathrm{du}$ cholestérol LDL et une diminution du cholestérol HDL, n'est retrouvé dans notre étude que chez $31,8 \%$. Cette faible proportion va dans le sens d'un contrôle plus régulier du bilan lipidique, comme le proposent Marant et al. (2008), car la répartition obtenue dans ce travail est susceptible de modifications au fil du temps, et surtout en fonction de l'équilibre glycémique.

\section{Conclusion}

Ce travail a démontré que les diabétiques de type 2 de Libreville au Gabon avaient un risque cardiovasculaire particulièrement élevé, en tenant compte de leur profile lipidique. Ce risque devrait amener à une amélioration de leur prise en charge, notamment par une utilisation plus fréquente des hypolipémiants et des antioxydants.

\section{REMERCIEMENTS}

Nous remercions le Dr Eric Baye pour sa participation au recrutement des malades.

\section{REFERENCES}

AFSSAPS. 2001. (Agence Française de Sécurité Sanitaire des Produits de Santé). La prise en charge thérapeutique du patient dyslipidémique. Recommandation de bonne pratique. Rev. Prat., 15(522): 58-62.

AFSSAPS (Agence Française de Sécurité Sanitaire des Produits de Santé). 2005. Prise en charge du patient dyslipidémique. Paris.

Boutouyriel P, Tropeano AS, Laurant S. 2005. Remodelage artériel dans le diabète. S.T.V., 17(1): 14-22.

De Geest B, Stengel D, Landeloos M, Lox M, Le Gat L, Collen D, Holvoet P, Ninio E. 2000. Effect of over expression of human apo A-1 in C57BL/6 and C57BL/6 apo-E deficient mice on 2 lipoprotein associated enzymes, plateletactivating factor acetyl hydrolase and paraoxonase. Comparison of adenovirusmediated human apo-A1 gene transfer and human apo A-1 transgenics. Arterioscl. Thromb. Vasc. Biol., 20: E6875.

Dehout F, Haumont S, Gaham N, AmoussouGuenou KA, Hermans MP. 2008. Metabolic syndrome in bantu subjects with type 2 diabetes from sub-saharan extraction. Prevalence, gender differences and HOMA hyperbolic product. Diabetes and Metabolic Syndrome. Clinical Research and Reviews, 2: 5-11.

Emma Ashton L, Fabien SD, Madeleine JB. 2000. Effect of meat replacement by Tofu on CHD risk factors including 
copper induced LDL oxidation. J. Am. Coll. Nutr., 19:761-767.

Eschwege E. 2005. Diabète de type 2: l'épidémie mondiale, données françaises. Officiel Santé, 29: 60-62.

Esterbauer H, Striegel G, Puhl H, Rotheneder M. 1989. Continuous monitoring of in vitro oxidation of human low-density lipoprotein. Free Radic. Res. Commun., 6: 67-75.

Fezeu L, Balkan B, Sobngwi E, Kengne AP, Vol S, Ducimetière $\mathrm{P}$, Mbanya JC. 2010. Waist circumference and obesityrelated abnormalities in French and Cameroonian adults: the role of urbanization and ethnicity. Int. J. Obes., 34(3): 446-53.

Fruchart JC, Sacks FM, Hermans MP, Asmann G, Brown WV, Ceska R, Chapman MJ, Dodson PM, Fioretto P, Ginsberg HN, Kadowaki T, Lablanche JM, Marx N, Plutzky J, Reiner Z, Rosenson RS, Staels B, Stock JK, Sy R, Wanner C, Zambon A, Zimmet P. 2008. The residual risk reduction initiative: a call to action to reduce residual vascular risk in dyslipidaemic patients. Diabetes Vasc. Dis. Res., 5: 319-335.

Ginsberg HN, MacCallum PR. 2009. The obesity, metabolic syndrome and type 2 diabetes mellitus pandemic: Part I. Increased cardiovascular disease risk and the importance of atherogenic dyslipidemia in persons with the metabolic syndrome and type 2 diabetes mellitus. J. Cardiometab. Syndr., 4(2): 113-119.

Grundy SM, Cleeman JI, Merz CNB, Brewer HB Jr, Clark LT, Hunninghake DB, Pasternak RC, Smith SC Jr, Stone NJ. 2004. Implications of recent clinical trials for the National Cholesterol Education Program Adult Treatment Panel III Guidelines. Arterioscl. Thromb. Vasc. Biol., 24: E149-155.

Hall V, Thomsen RW, Henriksen O, Lohse N. 2011. Diabetes in Sub-Saharan Africa
1999-2011: epidemiology and public health implications. A systematic review. BMC Public Health, 11: 564.

Hirano T, Ito Y, Saegura H, Yoshiro G. 2003. A novel and simple method for quantification of small and dense lowdensity lipoprotein. J. Lipid Res., 44: 2193-2201.

Klisiewics AM, Raal F. 2009. Sub-optimal management of type 2 diabetes mellitus. A local audit. JEMDSA, 14(1): 13-16.

Marant C, Domon I, Fosse S, Weill A, Simon D, Eschwege E, Varroud-Vial M, FagotCampagna A. 2008. French medical practice in type 2 diabetes: the need for better control of cardiovascular risk factors. Diabetes Metab., 34: 38-45.

Martin M, Hulley SM, Browner WW. 1986. Serum cholesterol, blood pressure and mortality: implications from a cohort of 361662 men. Lancet, 2: 933-936.

Mengesha AY. 2006. Lipid profile among diabetes patients in Gaborone, Botswana. S. Afr. Med. J., 96(2): 147-148.

Ngou-Milama E, Liamidi A, Makandja R, Methogo M, Moundounga B, Okomo B, Kombila P, Parra HJ. 1997. Valeurs de référence du cholestérol total, des triglycérides, de l'apolipoprotéine A1, de l'apolipoprotéine B et de la Lp(a) dans la population gabonaise. Etude sur 1020 sujets. Bulletin Médical d'Owendo, 18: 60-61.

Ntyonga-Pono MP, Nguemby Mbina C. 1996. Le diabète sucré à Libreville : Prévalence et perspective. Med. Afr. Noire, 43(7): 430-433.

Okafor CI, Fasanmade OA, Oke DA. 2008. Pattern of dyslipidemia among Nigerians with type 2 diabetes mellitus. Niger J. Clin. Pract., 11(1): 25-31.

Otero P, Bonet B, Herrera E, Rabano A. 2005. Development of atherosclerosis in the diabetic BALB/c mice. Prevention with vitamine $\mathrm{E}$ administration. Atherosclerosis, 182: 259-65.

Otieno CF, Mwendwa FW, Vaghela V, Ogola EN, Amavo EO. 2005. Lipid 
profile of ambulatory patients with type 2 diabetes mellitus at Kenyatta National Hospital, Nairobi. East Afr. Med. J., 8(12 suppl): S173-S179.

Rijks LG. 1995. Friedewald formula [Technical Brief]. Clin. Chem., 41: 761.

Sevum B, Mebrahtu G, Usman A, Mufunda J, Tewolde B, Haile S, Kosia A, Negassi E. 2010. Profile of patients with diabetes in Erytrea : results of first phase registry analyses. Acta Diabetol., 47(1): 23-7.

Simon D, Fagot-Campagna A, Eschwege E, Balkan B. 2005. Diabète : définition, dépistage et épidémiologie. In Traité de Diabétologie, Coordonnateur Grimaldi A. Médecine-Sciences Flammarion.

Sumner AE, Zhou J, Doumatey A, Imoisili OE, Amoah A, Acheampong J, Oli J, Johnson T, Adebamowo C, Ritimi CN. 2010. Low HDL-cholesterol with normal triglyceride levels is the most common lipid pattern in West Africans and African Americans with metabolic syndrome : implications for cardiovascular disease prevention. CVD Prev. Control, 5(3): 75-80.

Vezi ZB, Naidoo DP. 2005. Dyslipidaemia among black patients with type 2 diabetes. Cardiovasc. J. S. Af., 16(4): 194-198.

Vijian S, Hayward RA. 2003. Treatment of hypertension in type 2 diabetes mellitus: blood pressure goals choice of agents, and setting priorities in diabetes care. Ann. Intern. Med., 138: 593-602.

World Medical Association. 2004. Declaration of Helsinki. Ethical principles for medical research involving human being subjects; Helsinki.

Youmbissi TJ, Djoumessi S, Nouedoui C, Ndobo P, Meli J. 2001. Profil lipidique d'un groupe d'hypertendus Camerounais noirs Africains. Med. Af. Noire, 48(7): 305-314. 